\title{
Research on the Method for Computer-aided Pattern Design of Uygur's Headwear Based on OpenCV
}

\author{
Shuxian Liu \\ Information Science \& Engineering Institution \\ Xinjiang University \\ Urumqi Xinjiang, China \\ e-mail:1sx623@163.com
}

\author{
Xiaohua Li \\ Computer Science \& Technology Institution \\ Xinjiang Normal University \\ Urumqi Xinjiang, China \\ e-mail: 1xhxjnu@126.com
}

\begin{abstract}
Xinjiang Uygur's flower hat is famous all over the world, it leads the world of headwear of various nationalities within China by the variety of styles, the various ways of how to embroider pattern design, and exquisite manufacture. It is unusual even in national costumes art of the world. The purpose of this paper is to analyze Uygur's headwear, study the rules of Uygur's headwear pattern, analyze the characteristic of the basic pattern, research on the key technologies based on OpenCV for computer-aided pattern design of Uygur's headwear, explore the application of computer-aided design in traditional costume's pattern design, and to realize application exploration of computer-aided design in designing of costume and dress adornment.
\end{abstract}

\section{Keywords- Flower Hat; Pattern; OpenCV; Bézier Curves}

\section{UYGUR'S FLOWER HAT AND RULES OF PATTERN}

The variety of Xinjiang Uygur's flower hat's [1] has most closely related to history, economy, religion, culture, arts and crafts, custom, and phylogeny of national amalgamation. Xinjiang is an important transportation hub on the historic Silk Road, so after the Han Dynasty, Xinjiang becomes post of all kinds of economy and culture gathering. This comes into being the especial effect on the development of economy, culture, and art in Xinjiang.

The ancient western regions is the galaxy place of three great world religions, the bridge of Chinese and foreign cultural interaction, and it makes ancient civilization of Xinjiang has a species peculiar to this area and is differ from others. Uygurs has several migration in history and many reasons, Uygurs assimilates with other nationalities. Because of different of ethnic group, language, culture and custom, every nationality's culture and art leave print for Uygur's culture, it makes Uygur's culture and art become a mixture of different types, and Uygurs create gorgeous and much variety of industrial arts. The development of industrial arts is applied in real situations, and Xinjiang's hat is one of the outstanding examples.

In the model of flower hat [2], there are generally several types: quatrefoil (it is sewed with four piece of triangle materials), hat of five sides, goblets of hat, white and embroidered hat, white and geometric pattern hat, color cloth embroidered hat of dichromatism or four colors and high flower hat etc.

There are several types of flower hat pattern: layout with poppyhead composed by four symmetric rotundities, ellipses, squarenesses, long triangles, and anomalous formations, and the edge is four rectangles design; layout with four symmetric poppyhead of rotary pattern, and the edge is three series pattern; layout with arranged regularly points and spread; layout with cross symmetry, each piece has framework of cross, and plan pattern according to Tian-shaped, M-shaped or square; layout with circular poppyhead, and the edge is multilayer and different width of two series pattern; and layout with five group pattern as edge's pattern.

The pattern of flower hat has plant veins, such as megranate flower, peach blossom, apricot flower, chrysanthemum, peony, cotton, grape, fig, calliopsis, rose, lotus and so on; veins of natural phenomenon, for example, the hosts of heaven, snowflake; veins of animal, such as butterfly, fish; and all kinds of geometric pattern. As a part of cultural heritage, traditional manual pattern design [3,4] contains deep root of traditional culture, keeps the nature state of the national culture and the peculiar modes of their thought, and it has very high values in taste, culture, and application. With the increasing recognition of protection of traditional cultural heritage all the countries in the world, the protecation of traditional manual pattern design, especially research of the digit model has drawn more and more attention.

\section{BÉZIER CURVES}

A Bézier curve [5] is a parametric curve frequently used in computer graphics and related fields. Generalizations of Bézier curves to higher dimensions are called Bézier surfaces, of which the Bézier triangle is a special case. In vector graphics, Bézier curves are used to model smooth curves that can be scaled indefinitely. "Paths," as they are commonly referred to in image manipulation programs, are combinations of linked Bézier curves. Paths are not bound by the limits of rasterized images and are intuitive to modify. Bézier curves are also used in animation as a tool to control motion. Bézier curves are also used in the time domain, particularly in animation and interface design, e.g., a Bézier curve can be used to specify the velocity over time of an object such as an icon moving from A to B, rather than simply moving at a fixed number of pixels per step. When animators or interface designers talk about the "physics" or "feel" of an operation, they may be referring to the particular Bézier curve used to control the velocity over time of the move in question.

Bézier curves were widely publicized in 1962 by the French engineer Pierre Bézier, who used them to design automobile 
bodies. But the study of these curves was first developed in 1959 by mathematician Paul de Casteljau using de Casteljau's algorithm, a numerically stable method to evaluate Bézier curves.

The realization of computer-aided pattern design of Uygur's headwear by use of de Casteljau's algorithm in this paper.

If P0, P02, P2are three different points on the parabola, two tangents through point $\mathrm{P} 0$ and point $\mathrm{P} 2$ cut at point $\mathrm{P} 1$, the tangent through point $\mathrm{P} 02$ cut line $\mathrm{P} 0 \mathrm{P} 1$ at point $\mathrm{P} 01$ and cut line $\mathrm{P} 2 \mathrm{P} 1$ at point $\mathrm{P} 11$, and as follows proportion come into existence:

$$
\frac{P_{0} P_{0}^{1}}{P_{0}^{1} P_{1}}=\frac{P_{1} P_{1}^{1}}{P_{1}^{1} P_{2}}=\frac{P_{0}^{1} P_{0}^{2}}{P_{0}^{2} P_{1}^{1}}
$$

It is called three tangent theorem of parabola. Such as "Fig. 1 " is a Bézier curve.

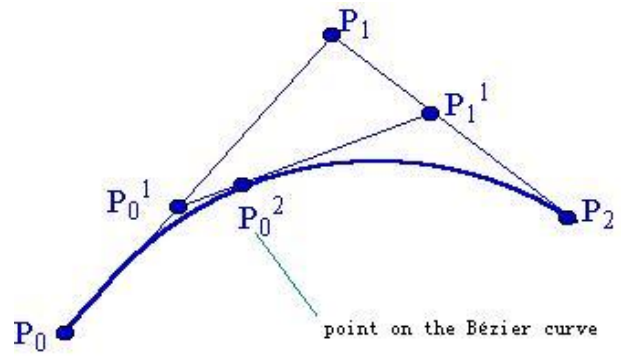

Figure 1. Bézier curve.

It is imported a parametert when point $\mathrm{P} 0$ and point $\mathrm{P} 2$ are fixed, and let above ratio is $\mathrm{t}:(1-\mathrm{t})$, that is:

$$
\begin{aligned}
& P_{0}^{1}=(1-t) P_{0}+t P_{1} \\
& P_{1}^{1}=(1-t) P_{1}+t P_{2} \\
& P_{0}^{2}=(1-t) P_{0}^{1}+t P_{1}^{1}
\end{aligned}
$$

t varies from 0 to 1 , the first and second formula separately shows the first and second side which control the two side shape, they are called linear Bézier curves. And let the first and second formula in the third formula, it can be written as:

$$
P_{0}^{2}=(1-t)^{2} P_{0}+2 t(1-t) P_{1}+t^{2} P_{2}
$$

$\mathrm{t}$ varies from 0 to 1 , it is quadratic Bézier curve, which is the path traced by the points P0, P1, and P2. And it can be interpreted as the linear interpolant of corresponding points on the linear Bézier curves from P0 to P1 and from P1 to P2 respectively. Bézier curves can be defined for any degree $n$. A recuresive definition for the Bézier curve of degree $n$ expresses it as a point-to-point linear combination of a pair of corresponding points in two Bézier curves of degree n-1.

$$
P_{0}^{n}=(1-t) P_{0}^{n-1}+t P_{1}^{n-1} \quad t \in[0,1]
$$

The recuresive compute formula for Bézier curve can be written as:

$$
P_{i}^{k}= \begin{cases}P i & k=0 \\ (1-t) P_{i}^{k-1}+t P_{i+1}^{k-1} & k=1,2, \cdots, n \quad t=0,1, \cdots, n-k\end{cases}
$$

It is De Casteljau's algorithm.

\section{OPENCV}

OpenCV (Open Source Computer Vision) is a library of programming functions for real time computer vision.

OpenCV [6] is released under a BSD license, it is free for both academic and commercial use. It has $\mathrm{C}++, \mathrm{C}$, Python and soon Java interfaces running on Windows, Linux, Android and Mac. The library has $>2500$ optimized algorithms (see figure below). It is used around the world, has $>2.5 \mathrm{M}$ downloads and $>40 \mathrm{~K}$ people in the user group. Uses range from interactive art, to mine inspection, stitching maps on the web on through advanced robotics.

OpenCV has a modular structure, which means that the package includes several shared or static libraries. The following modules are available:

- core - a compact module defining basic data structures, including the dense multi-dimensional array Mat and basic functions used by all other modules.

- imgproc - an image processing module that includes linear and non-linear image filtering, geometrical image transformations (resize, affine and perspective warping, generic table-based remapping), color space conversion, histograms, and so on.

- video - a video analysis module that includes motion estimation, background subtraction, and object tracking algorithms.

- calib3d - basic multiple-view geometry algorithms, single and stereo camera calibration, object pose estimation, stereo correspondence algorithms, and elements of $3 \mathrm{D}$ reconstruction.

- features $2 \mathrm{~d}$ - salient feature detectors, descriptors, and descriptor matchers.

- objdetect - detection of objects and instances of the predefined classes (for example, faces, eyes, mugs, people, cars, and so on).

- $\quad$ highgui - an easy-to-use interface to video capturing, image and video codecs, as well as simple UI capabilities.

- gpu - GPU-accelerated algorithms from different OpenCV modules.

... some other helper modules, such as FLANN and Google test wrappers, Python bindings, and others.

\section{CONTOUR DETECTION AND EDGE DETECTION}

\section{A. Contour Detection}

Contour detection has been a field of large interest in recent years. Kass et al. introduced the active contour model ("snakes") to find salient image contours. For this approach, the contour is represented as a chain with a fixed number of vertices. The modeling of the contour is made through differentiable energy functions measuring local smoothness. The observed image is introduced through an additional energy function incorporating the pixel intensities or their gradients. Discrete dynamic programming has been used for finding the optimal contour. In 
this case, the requirement of differentiability of the energy functions can be relaxed and "hard constraints", such as a minimum adjacent internode distance, can be introduced during the minimization process.

In Friedland and Adam a centerpoint inside the contour and a number of angularly equispaced radii were defined. The contour, assumed to be star-shaped, was then represented by the lengths along the radii from the centerpoint. A set of energy functions were constraining the contour, and the optimization algorithm used was based on simulated annealing. By restricting the number of possible lengths along each radius, this representation also falls into the one used for the active contour model.

A parametric model based on elliptic Fourier decomposition of the contour was formulated by Staib and Duncan. They assumed a one-to-one and continuous mapping between contour curves in image space and their parameterization. The parameters of the (truncated) elliptic Fourier series were regarded as random variables and boundary finding was formulated as an optimization problem in a Bayesian framework using the MAP estimate.

\section{B. Edge Detection}

Edge detection is the name for a set of mathematical methods which aim at identifying points in a digital image at which the image brightness changes sharply or, more formally, has discontinuities. The points at which image brightness changes sharply are typically organized into a set of curved line segments termed edges. The same problem of finding discontinuities in 1D signals is known as step detection and the problem of finding signal discontinuities over time is known as change detection. Edge detection is a fundamental tool in image processing, machine vision and computer vision, particularly in the areas of feature detection and feature extraction.

The purpose of detecting sharp changes in image brightness is to capture important events and changes in properties of the world. It can be shown that under rather general assumptions for an image formation model, discontinuities in image brightness are likely to correspond to:

- discontinuities in depth,

- discontinuities in surface orientation,

- changes in material properties and variations in scene illumination.

\section{THE THREAD HOW TO DRAWING PATTERN}

The pattern of flower hats is composed of some basic patterns, and those basic patterns make up of smooth and clear lines.

Based on rules of flower hats' pattern, several questions that are needed to consider during the realization of drawing: how to store pattern, whole pattern or analysis to draw, how to get the whole pattern by drawing of analysis, and so on. Because the patterns mostly are symmetric, each complicated design may be regard as synthesis of some basic patterns, basic patterns are composed of smooth curves, and curves make of many points. But the workload drawing every points to paint the curve is very great.

The thread of this paper is: first, to analyze the original pattern, and to realize contour detection, second, to complete edge detection, third, to clip and get curves, fourth, to draw the curves by algorithm of Bézier curves, fifth, to synthesize basic designs with curves by algorithms of mirror, rotation, and synthesis, finally, to synthesize the whole pattern with basic designs. To summarize the point is to realize the drawing by "contour detection edge detection clip curves drawing synthesis".

BMP(Bitmap File) is used in this paper to store patterns. And the process which shows BMP is:

- $\quad$ apply memory to store bitmap file

- $\quad$ read the bitmap file in memory that applied

- create display of bitmap by using related functions

It takes the original pattern that showed in Fig. 2. for an example to explain the idea how to draw flower hats' pattern.

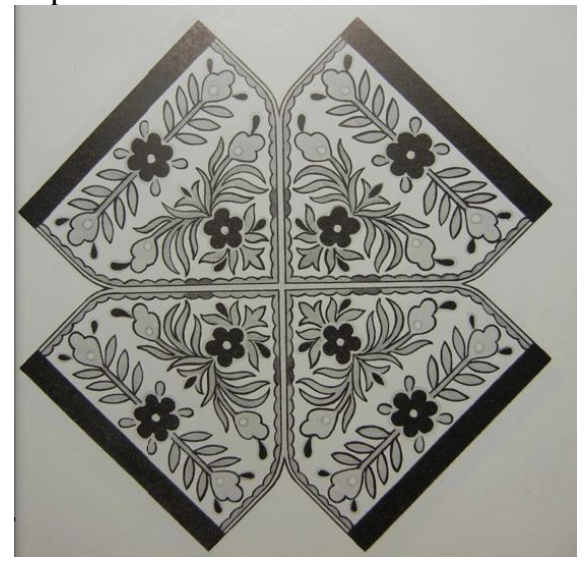

Figure 2. original pattern.

First, analyze the original pattern, complete contour detection, it's showed in Fig. 3.

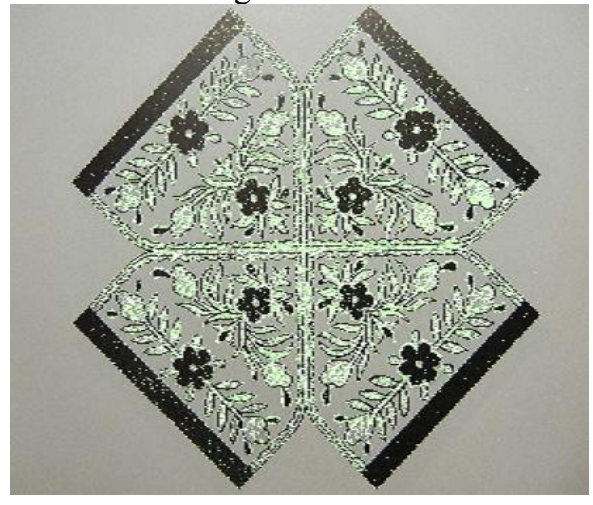

Figure 3. contour detection.

Second, to complete edge detection, it's showed in Fig. 4. 


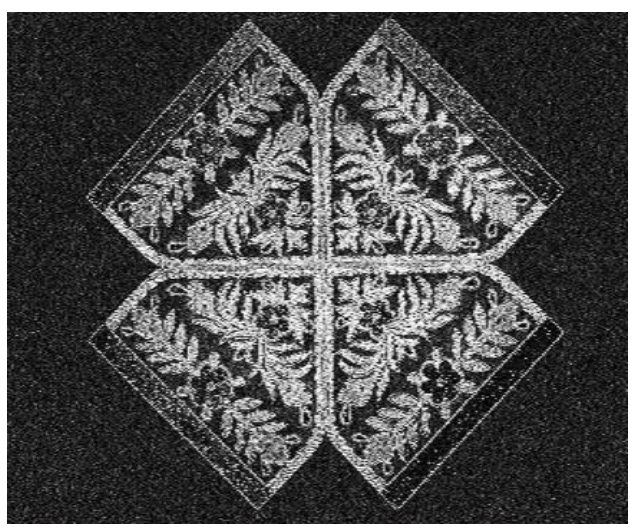

Figure 4. edge detection.

Finally, draw the basic patterns by clipping to get curves and drawing the curves by algorithm of Bézier curves, it's showed in Fig. 5. The application of Bézier curves in design is using De Casteljau algorithm.

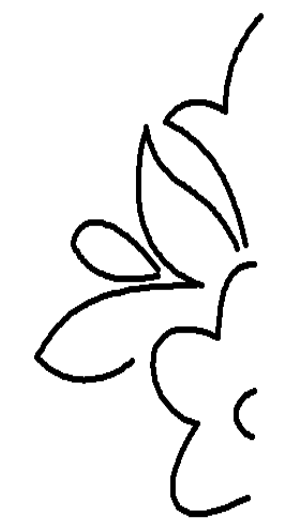

Figure 5. the pattern of drawing

The designs are beautiful, curves are smooth, and true to the original patterns. So it is feasible to realize the drawing of flower hat patterns by basic idea of Bézier curves and relative algorithms. And it verifies further the method which used in this paper is correct.

\section{CONCLUSION}

It introduces the rules of Uygur's headwear pattern, provides a method of drawing by "contour detection $\rightarrow$ edge detection $\rightarrow$ clip $\rightarrow$ curves drawing $\rightarrow$ synthesis", and gives an example to explain the method. Thus it shows the method that draw Uygur's flower hat pattern by Bézier curves and OpenCV is correct.

\section{ACKNOWLEDGMENT}

The project Jointly Funded by College and Institute of Xinjiang University, "Research on the Technologies for Computer-aided Pattern Design of Uygur's Headwear" (ID:XY110120)

\section{REFERENCES}

[1] Luo Huizhen, "Cultural Survery of Uygur's Flower Hat in Xinjiang," Society and Economy of Xinjiang. vol. 3, 1998, pp.72-75.

[2] Wu Shining, "the art worn on the head-Uygur's flower hat", Art Observer, vol. 123, 2009, pp.115.
[3] Kaplan CS, Salesin DH, "Islamic star patterns in absolute geometry," ACM Transactions on Graphics, vol. 23, No. 2, 2004, pp. 97-119.

[4] Karam H, Nakajing M. , "Islamic symmetric pattern generation based on group theory", Washington DC, 1999, pp. 112-114.

[5] Cai Feilong, Peng Ren, Yu Jinhui, "Analysis and Synthesis of Peking Opera Facial Make-Ups,"Journal of Computer-Aided Design \& Computer Graphics, vol. 21, No. 8, Aug., 2009, pp. 1092-1097.

[6] http://opencv.org/ 2013.5.25. 